\title{
Diacronie
}

Studi di Storia Contemporanea

$N^{\circ} 28,4 \mid 2016$

La voce del silenzio

\section{Christina Garsten, Bo Rothstein, Stefan Svallfors, Makt utan mandat: de policyprofessionella $i$ svensk politik}

Potenza senza mandato: il professionista della politica nella politica svedese

\section{Monica Quirico}

\section{OpenEdition}

\section{Journals}

Edizione digitale

URL: http://journals.openedition.org/diacronie/4494

DOI: $10.4000 /$ diacronie.4494

ISSN: 2038-0925

\section{Editore}

Association culturelle Diacronie

Notizia bibliografica digitale

Monica Quirico, «Christina Garsten, Bo Rothstein, Stefan Svallfors, Makt utan mandat: de

policyprofessionella i svensk politik », Diacronie [Online], № 28, 4 | 2016, Messo online il 29 décembre 2016, consultato il 24 septembre 2020. URL : http://journals.openedition.org/diacronie/4494 ; DOI : https://doi.org/10.4000/diacronie.4494 


\title{
Diacronie
}

N. 28 | 4|2016 La voce del silenzio: intelligence, spionaggio e conflitto nel XX secolo

\section{8/}

\section{PANORAMICA: Svezia e Finlandia 2015}

\author{
Kasper BRASKÉN, Monica QUIRICO *
}

\author{
SAARELA, Tauno, Finnish Communism Visited, Helsinki, The Finnish \\ Society for Labour History, 2015, 233 pp. \\ a cura di Kasper BRASKÉN \\ traduzione di Alessandro SALVADOR
}

Il volume raccoglie dieci articoli dell'autore, Tauno Saarela, docente di Storia Politica all'Università di Helsinki. Si tratta di una selezione di scritti pionieristici sulla storia del comunismo finlandese. Il movimento comunista finlandese è stato uno dei più grandi in Europa ed era plasmato in buona parte dalla sua vicinanza, anche geografica, con l'Unione Sovietica. La storia del comunismo finlandese include anche molte peculiarità che la rendono un caso particolarmente interessante nel panorama internazionale. Gli articoli contenuti in questa antologia ricoprono un periodo che va dalla formazione e sviluppo del comunismo finlandese, nel 1918, fino agli anni Sessanta, con un'enfasi particolare sul periodo tra le due guerre. L'evoluzione del fenomeno in Finlandia è analizzata, lungo tutto il volume, in relazione al movimento comunista internazionale e con una specifica attenzione per le organizzazioni russe, scandinave e americane. Tra i temi centrali del libro è inclusa l'analisi del comunismo culturale che include, tra gli altri, un articolo sulla gioventù comunista e la musica popolare dopo la Seconda guerra mondiale. Viene anche discussa la nascita del culto della personalità nel comunismo, anche con una interessante prospettiva comparativa tra il leader comunista Otto Ville Kuusinen e il generale Carl Gustaf Emil Mannerheim. Saarela offre anche interessanti contributi al dibattito sulla stalinizzazione, la bolscevizzazione del movimento comunista e le tensioni tra il contesto nazionale e quello internazionale. Paradossalmente, come mostra Saarela, la stalinizzazione del comunismo finlandese non inizia che nel 1943, dopo la dissoluzione dell'Internazionale 
Comunista. Riassumendo, le prospettive internazionali, transnazionali e comparative di questo volume lo rendono un contributo di grandissimo interesse per la ricerca internazionale. Inoltre, offre la possibilità di accesso alle ricerche più recenti, solitamente pubblicate in finlandese, anche ad un pubblico anglofono.

KOIVISTO, Hanne, PARIKKA, Raimo (eds.), iNO PASARÁN! Espanjan Sisällissodan Kulttuurihistoriaa [¿NO PASARÁN! A Cultural History of the Spanish Civil War], Helsinki, The Finnish Society for Labour History, 2015, 464 pp.

a cura di Kasper BRASKÉN

traduzione di Alessandro SALVADOR

L'antologia No Pasaran contiene 16 contributi scientifici che si occupano della storia della guerra civile spagnola. Gli articoli erano stati inizialmente presentati ad una conferenza sulla storia culturale della guerra civile spagnola organizzata nell'aprile del 2014 presso l'Università di Turku, in Finlandia. I capitoli del libro si occupano della guerra civile secondo diverse prospettive interdisciplinari: politica, cultura, legge, letteratura, religione e arte. Il libro affronta la guerra civile come guerra totale e analizza la vasta guerra di propaganda che fu combattuta da artisti di alto livello, autori e giornalisti, soprattutto quelli schierati sul fronte repubblicano. Due capitoli analizzano la Guerra civile anche dal punto di vista dei ribelli e dei loro alleati italiani. Seguendo la grande tradizione della storia culturale, diversi capitoli si occupano degli aspetti visuali, sonori, olfattivi, gustativi ed emozionali della guerra civile, estendendo così le narrative tradizionali. Tra gli altri, le fotografie di Robert Capa, Grenada, Grenada, Grenada moja di Roman Karmen e la produzione letteraria di André Malraux sono tra i casi analizzati. Alcuni capitoli si occupano delle reazioni finlandesi nei confronti della Guerra civile spagnola, inclusa la solidarietà degli intellettuali di sinistra, della classe operaia e dei volontari finlandesi nel conflitto. In conclusione, l'antologia offre un contributo importante alla storia della guerra civile spagnola nel contesto della storiografia finlandese e rilancia la ricerca internazionale sul tema in una prospettiva storico-culturale. 


\section{LIEDMAN, Sven Eric, Karl Marx. En biografi [Karl Marx. Una biografia], Stockholm, Bonnier, 2015,818 pp.}

a cura di Monica QUIRICO

Tra i maggiori storici svedesi delle idee politiche, Liedman ha lavorato, nel corso della sua lunga carriera, a saggi e traduzioni dedicati a Marx ed Engels e al marxismo. L’imponente biografia (più di 800 pagine) che ora presenta può quindi essere considerata il coronamento di un costante interesse per tale ambito di studi. Tuttavia, ciò che preme all'autore, accanto alla ricostruzione scrupolosa della produzione politico-intellettuale di Marx (le grandi opere così come i contributi meno noti), è il confronto con l'(ab)uso che delle sue teorie è stato compiuto, e, insieme, con l'inatteso revival delle sue analisi, in tempi di crisi economica - o, come direbbe il Nostro, di crisi del capitalismo. In tale labirintico intreccio tra il messaggio (prettamente ottocentesco quanto a contesto e destinatari) trasmesso dall'opera marxiana e le interpretazioni che vi si sono sovrapposte a partire dal 1917, si destreggia Liedman, sfidando le tradizionali letture che fanno di Marx un teorico dell'anti-individualismo, identificando contemporaneamente nel capitalismo la formazione sociale anti-deterministica per eccellenza. In breve, Liedman mette in guardia dal Marx che abbiamo imparato a conoscere nel Novecento, troppo contaminato dal dogmatismo (tanto quello amico quanto quello ostile), invitandoci a riscoprire il Marx delle origini: a prescindere dall'intrinseco valore filologico dell'operazione, è questo ritorno a Marx, nel suo tempo storico, che ha ancora molto da dire a chi non si accontenta delle promesse, ormai sempre più logore, del neoliberalismo.

GARDELL, Mattias, Raskrigaren. Seriemördaren Peter Mangs [Raskrigaren. Il serial killer Peter Mangs], Stockholm, Leopard, 2015, 439 pp.

a cura di Monica QUIRICO

Tra il 1991 e il 1992 John Ausonius, un giovane uomo di origini tedesche, colpisce con un fucile a mirino laser (da qui il soprannome "uomo laser") undici persone, tra Stoccolma e Uppsala; una rimane uccisa, mentre altre riportano invalidità permanenti. Il comune denominatore delle vittime è il loro aspetto non europeo. La storia si ripete sempre due volte: nel secondo caso affiora, pur nella tragedia della violenza xenofoba, una componente di farsa. Nel 2012 Peter Mangs viene condannato per due omicidi, quattro tentati omicidi e altri atti di violenza perpetrati a Malmö tra il 2003 e l'anno 
dell'arresto, il 2010 - di nuovo, a danno di immigrati, nonché, a differenza di Ausonius, di ebrei, rom e "traditori della razza". Mattias Gardell, docente di storia della religione all'Università di Uppsala, ricostruisce il mondo rovesciato di Mangs, insistendo su affinità e differenze tra il suo percorso e quello di Anders Behring Breivik, ma soprattutto sul fallimento, culturale prima ancora che operativo, del sistema giudiziario e dei media, che, anche dopo l'arresto, non vogliono accettare la realtà: il movente del serial killer era, sic et simpliciter, il razzismo; per quasi un decennio Mangs ha seminato il panico in una delle principali città della Svezia contando sul fatto che nessuno avrebbe mai sospettato di un uomo come tanti, sobrio, mite - e di etnia svedese; un vero svedese, anzi, proprio come gli esponenti del potere giudiziario e mediatico. Così è stato.

\section{ODENSTEN, Per, Människoütarens skugga [L'ombra del cannibale], Stockholm, Norstedts, 2015, 233 pp.}

a cura di Monica QUIRICO

Un prigioniero e il suo carceriere. Il primo, un noto intellettuale condannato per le sue idee "sovversive", è in precarie condizioni di salute dopo la detenzione in un altro carcere; non è mai nominato, ma il lettore riconosce immediatamente la figura di Antonio Gramsci. Il secondo, Leon, analfabeta fino all'età di venticinque anni, è l'io narrante del romanzo; iscritto, come tutti i dipendenti pubblici, al partito al potere (anch'esso mai nominato), non ha nulla di personale contro il prigioniero: semplicemente, deve fare la sua parte e dividere con lui l'isolamento in cui entrambi si ritrovano condannati all’interno della "Capsula 4", una sorta di moderno Panopticon. Il loro è un rapporto fatto di diffidenza - imposta dalle regole del gioco - ma anche, soprattutto da parte di Leon, di curiosità intellettuale e insieme personale verso un uomo demonizzato come terrorista e al quale pure il carceriere accetta di fare da intermediario nei messaggi scritti che l'uomo scambia, attraverso un divisorio, con una parente sordomuta venuta a visitarlo. Sciogliendo la narrazione dal riferimento stretto all'epoca e alla società di Gramsci, Odensten, con uno stile asciutto, quasi scarno, invita il lettore a riflettere sui costi di una società dominata dalla paura (dell'Islam, ad esempio). 
GARSTEN, Christina, ROTHSTEIN, Bo, SVALLFORS, Stefan, Makt utan mandat: de policyprofessionella i svensk politik [Potenza senza mandao: il professionista della politica nella politica svedese], Stockholm, Dialogos, 2015, 313 pp.

a cura di Monica QUIRICO

Tre influenti studiosi - l'antropologa sociale Garsten, il politologo Rothstein e il sociologo Svallfors - presentano in questo volume i risultati della loro pluriennale ricerca sulle peculiarità dei "professionisti della politica" in Svezia. L'espressione designa quei soggetti che, pur svolgendo ruoli politici di primo piano (come spin doctor e consulenti a vario titolo, lobbisti, nonché think tank e altri organismi pseudoscientifici), non hanno ricevuto alcuna investitura democratica. Benché la Svezia non sia ancora arrivata, a differenza dell'Italia, ad avere un Primo ministro non eletto dal popolo, il volume rivela nondimeno che neppure la patria della socialdemocrazia ha potuto o voluto disporre di antidoti a una tendenza che sta intaccando a livello globale la natura della democrazia, e che ciò nonostante rimane poco indagata, soprattutto nell'Europa meridionale. La ricerca di Garsten, Rothstein e Svallfors merita di essere segnalata perché rappresenta, nel contesto svedese, la prima mappatura, condotta anche attraverso un'ampia mole di interviste, di un lato della politica invisibile agli occhi dei comuni cittadini e che nondimeno condiziona sempre più l'azione dei partiti e delle istituzioni. 


\section{* Gli autori}

Kasper Braskén è ricercatore post-doc presso la Åbo Akademi University di Turku, Finlandia. È uno storico specializzato in storia tedesca, transnazionale e dei movimenti sociali. Braskén è coeditore di H-Socialisms e autore di diversi articoli sulla solidarietà internazionale, sul comunismo e sull'antifascismo. La sua tesi di dottorato è stata pubblicata nel 2015 col titolo: The International Worker's Relief, Communism and Transnational Solidarity: Willi Münzenberg in Weimar Germany (Basingstoke, Palgrave Macmillan, 2015).

URL: < http://www.studistorici.com/progett/autori/\# Brasken >

Monica Quirico, honorary research fellow presso l'Istituto di storia contemporanea della Södertörn University di Stoccolma, attualmente è affiliata all'Istituto per la storia della Resistenza di Torino, dove partecipa al progetto di ricerca internazionale "The Politics of Military Loss in Contempoary Europe", coordinato dall'Università di Stoccolma e finanziato dal Consiglio nazionale della Ricerca svedese (VR). Si è occupata di storia del liberalismo, totalitarismo e storia e società svedese. Ha pubblicato, oltre a numerosi contributi per volumi e riviste, italiani e stranieri, L“Unione Culturale” di Torino: antifascismo, utopia e avanguardie nella città-laboratorio (1945-2005), Roma, Donzelli, 2010; Tra utopia e realtà. Olof Palme e il socialismo democratico, traduzione e cura di M. Quirico, Roma, Editori Riuniti - University Press, 2009; Il socialismo davanti alla realtà. Il modello svedese (1990-2006), Roma, Editori Riuniti, 2007; Collettivismo e totalitarismo. Hayek e Michael Polanyi (1930-1950), Milano, FrancoAngeli, 2004.

URL: < http://www.studistorici.com/2015/o4/18/monica_quirico/ >

\section{Per citare questo articolo:}

BRASKÉN, Kasper, QUIRICO, Monica, «Panoramica: Svezia e Finlandia 2015», Diacronie. Studi di Storia Contemporanea : La voce del silenzio: intelligence, spionaggio e conflitto nel XX secolo, 29/12/2016,

URL:<http://www.studistorici.com/2016/12/29/scandinavia_numero_28/ >

Diacronie Studi di Storia Contemporanea 3 www.diacronie.it

Risorsa digitale indipendente a carattere storiografico. Uscita trimestrale. redazione.diacronie@hotmail.it

Comitato di redazione: Jacopo Bassi - Luca Bufarale - Antonio César Moreno Cantano - Deborah Paci - Fausto Pietrancosta - Alessandro Salvador - Matteo Tomasoni - Luca Zuccolo

Diritti: gli articoli di Diacronie. Studi di Storia Contemporanea sono pubblicati sotto licenza Creative Commons 3.0. Possono essere riprodotti e modificati a patto di indicare eventuali modifiche dei contenuti, di riconoscere la paternità dell'opera e di condividerla allo stesso modo. La citazione di estratti è comunque sempre autorizzata, nei limiti previsti dalla legge. 